\title{
Economic Dispatch Problem in Power System Using Hybrid PSO and Enhanced Bat Optimization Algorithm
}

\author{
Mr. Rupam Gupta Roy \\ Department of Electronics and Instrumentation Engineering \\ National Institute of Technology, \\ Agartala, Tripura, India \\ royrupamgupta@gmail.com
}

\begin{abstract}
This paper presents a competent meta-heuristic algorithm, named, Particle Swarm Optimization- Enhanced Bat optimization (PSO-EBO) algorithm to solve the optimal operating approach of the ELD issue. The developed technique integrates two fundamental ideas. Initially, the swarm behavior of the particle is exploited to find the optimal values of the EBO technique. To illustrate the developed method performance, it is used on large, medium, and small, scale test systems to solve the ELD issues of 13-unit, 40-unit and160-unit systems. Moreover, the comparative results are performed to examine the effectiveness of the developed PSO-EBO algorithm with the existing Grey Wolf Optimization (GWO), Artificial Bee Colony (ABC), Whale Optimization Algorithm (WOA) and Differential Evolution (DE) algorithms. Finally, the experimentation outcomes obviously propose that the developed PSO-EBO algorithm can find superior solutions regarding the computational time and fuel cost than conventional methods.
\end{abstract}

Keywords: Meta-Heuristic; Economic Dispatch; Constraints; Fuel Cost; Power System

\begin{tabular}{ll}
\multicolumn{2}{l}{ Nomenclature } \\
\hline Abbreviations & Descriptions \\
\hline ELD & Economic Load Dispatch \\
POZ & Prohibited Operating Zones \\
BA & Bat Algorithm \\
ED & Economic Dispatch \\
PSO & Particle Swarm Optimization \\
SSM & Simplex Search Method \\
WOA & Whale Optimization Algorithm \\
AGWO & Ameliorated Grey Wolf Optimization \\
ABC & Artificial Bee Colony \\
VPE & Valve Point Effects \\
PPSO & Phasor PSO \\
GA & Genetic Algorithm \\
EPLD & Economic Power Load Dispatch \\
HAAA & Hybrid Artificial Algae Algorithm \\
DP & Dynamic Programming \\
MMS & Multiple Mutation Strategies \\
ELDP & Economic Load Dispatch Problem \\
ACS & Artificial Cooperative Search \\
ADE & Differential Evolution \\
IGA & Improved GA \\
RRL & Ramp Rate Limits \\
\hline
\end{tabular}

\section{Introduction}

In the economy of the country, the electrical power system plays a significant role and it is a large interconnected system. In favor of the competent and consistent operation of such a large interconnected power system, it needs appropriate evaluation and technique to operate such a system cost-effectively. In 
the electrical power system, the ELD issue is a significant optimization task and study of ELD aids to operate power systems economically using a competent method and present power without any disruption. The ELD is an online process of assigning generation between available generating units to reduce total generation cost and assure equality and inequality constraints. Gradually civilization raises the electricity demand raises in a similar ratio. For fulfillment of load demand, huge numbers of thermal power plants have deployed and the capability of coal burnt raises. Owing to burning great numbers of coal produced numerous toxic gases such as sulphur dioxide, nitrogen oxides, and carbon dioxide, at thermal power plants, and contaminate the environment [1].

Pollution is destructive for living creatures and the environment. Environmental pollution rises global warming and harms the Ozone layer. Hence in current developments, it is necessary to produce power by reducing cost and reduce pollutant environmental emissions. The research of ELD aids to produce power on the least amount of cost and too minimize the environmental emission effects [9].

ELD is the optimization of distributed generation, owing to the subsistence of POZ, VPE, and RRL, and multiple fuel options, the unit's power utilization characteristic function, is a discontinuous function and a non-differential nonlinear. Additionally, the loss of the transmission line is predictable in stable circumstances of the power system. Hence, while realistic unease of ELD issues is contemplated, a complex optimization issue about high dimensionality, non-differentiability, nonlinearity, and multiconstraint is created and requires resolving.

For numerous years, to resolving ED issues the traditional techniques of optimization have been the only techniques. System operators possess to make simpler/disregard practical and technical constraints of units and the network, because of the limits of the traditional techniques. Such simplifications split into two categories [10]. One is related to the accurateness of cost model of generating units particularly for diverse kinds of fuels or to regard as VPE. An additional associated with network topology, also limited or unnoticed to taking into consideration merely the transmission network loss [2].

Day by day the power demand rises and the cost of the fuel for power generation is increasing maximum, as a result, it turns out to be obligatory to reduce operational costs and to attain consistent operations of power generation systems. An optimal load dispatch increases the energy ability of thermal units, raises consistency of the system, and minimizes production cost. Amongst dedicated generating units, EPLD shares the load demand on a power system to achieve the economy of operation in a definite system and operational constraints [11]. Fundamentally, EPLD is a nonlinearly constrained optimization issue, possessing nonlinear cost characteristics, and inequality and equality constraints [3]. The investigation was concentrated to attain a global solution of an optimization issue by using exploitation and exploration processes integrating complex constraints. The derivative-based approaches like programming, lambda iteration process, gradient techniques, linear quadratic programming, Lagrangian multiplier technique, classical methods resolving coordination equations, were used to resolve the EPLD issue [4], in contemporary generating units possessing multi-valve steam turbines.

The main contribution of this works exploits a competent meta-heuristic hybrid PSO and EBO algorithm named PSO-EBO to solve the four kinds of non-convex ELD issues to investigate search space much high effectually to overcome the difficulties of other approaches. Because of the excellent swarm behavior of the developed PSO approach, solutions do not stick to local optimum points in complex optimization issues such as non-convex complex ELD issues. Finally, the performance analysis is performed to shows the efficiency of the developed algorithm.

\section{Literature Review}

In 2018, Diljinder Singh and J.S. Dhillon [1] developed the AGWO technique which integrates the heuristics of the GWO technique, using an arbitrary exploratory heuristic technique for enhanced development to solve the ELD problem utilizing non-smooth cost functions possessing VLE, power generation limits. Finally, the outcomes expose that the developed method was effectual to search the optimum generation schedule whereas fulfilling constraints.

In 2019, Maedeh Gholamghasemi et al [2], developed an enhanced particle swarm optimization algorithm named PPSO that alternates PSO control parameters about a phasor angle to resolve diverse kinds of non-convex and convex/non-smooth ELD issues in diverse benchmark power systems such as 10unit system taking into consideration of multiple fuels without/with VPE, 15-unit system taking into consideration of POZ and transmission losses without/with ramp rate limits.

In 2018, Mohit Kumar and J.S. Dhillon [3], presented an HAAA that hybridizes the AAA and SSM to resolve the ELD problem. The large, medium, and small electric power test systems were embarked on to examine the applicability of the developed technique. Finally, outcomes promote that developed method was better than the AAA regarding the computation time, exploitation capability, and global search capability whereas implemented to resolve both conventional functions and ELD problems. 
In 2019, S. Hr. Aghay Kaboli and Abdullah K. Alqallaf [4], focused on the emergent of the ACS optimization technique to resolve ED problems more accurately using minimum complexity than other meta-heuristic optimization techniques. The proposed method was a lately proposed two population search method based on the coevolution procedure with a high probability to find the optimal solution.

In 2019, Qiang Zhang et al [5], proposed a self-ADE method combining with MMS for the ELD issues. To enhance the exploitation and exploration abilities of the conventional DE approach, ADE-MMS has 3 additions to DE. Initially, 4 kinds of highly developed vectors produced by the diverse techniques were used in mutation schemes. Subsequently, a self-adaptable chosen method for multiple mutation schemes was deployed in iterations.

In 2017, Yizi Shang et al [6], developed a practical GA-based solution to solve ELDP and additional evaluates Improved GA (IGA) performance using that of DP. Particularly, their performance was expansively estimated regarding addressed ELDP during a case study of 26 turbines in the three Gorges Hydropower Plants with a focal point regarding the computation accurateness, computation time, and technique stability.

\section{Problem Formulation of ELD}

The present formulation treats ELD issues its objective is to minimize total generation cost when fulfilling several equality and inequality constraints to assign optimal generation between power generating units.

\subsection{Objective model}

The most important objective to solve the ELD issue is to reduce total generation cost to convene load demand of a power system over a few appropriate stages when fulfilling diverse constraints. The cost of fuel can be approximated using a quadratic function of generator power output. The cost of fuel for $\mathrm{i}^{\text {th }}$ generating units possessing quadratic cost function is stated in eq. (1).

$$
\mathrm{F}_{\mathrm{i}}=\mathrm{e}_{\mathrm{i}} \mathrm{p}_{\mathrm{gi}}^{2}+\mathrm{f}_{\mathrm{i}} \mathrm{p}_{\mathrm{gi}}+\mathrm{g}_{\mathrm{i}}
$$

In eq. (1), $e_{i} ; f_{i} ; g_{i}$ indicates fuel cost coefficients of the $i^{\text {th }}$ unit and $n$ indicates the number of generators. Nevertheless, practical ELD generally does not possess smooth cost functions. Rather, because of the VPE, their cost functions at all times possess non-differentiable points. Generally, sinusoidal functions are augmented to quadratic cost functions to indicate the cost function of practical generators. The generation units cost function using valve-point loading is indicated in eq. (2).

$$
\mathrm{F}_{\mathrm{i}}=\mathrm{e}_{\mathrm{i}} \mathrm{p}_{\mathrm{gi}}^{2}+\mathrm{f}_{\mathrm{i}} \mathrm{p}_{\mathrm{gi}}+\mathrm{g}_{\mathrm{i}}+\left|\mathrm{a}_{\mathrm{i}} \times \sin \left(\mathrm{f}_{\mathrm{i}} \times\left(\mathrm{p}_{\mathrm{gi}}^{\min }-\mathrm{p}_{\mathrm{gi}}\right)\right)\right|
$$

In eq. (2), $p_{\mathrm{gi}}^{\min }$ indicates the least power generation limits of the $i^{\text {th }}$ generator and $\mathrm{a}_{\mathrm{i}}$ and $\mathrm{f}_{\mathrm{i}}$ indicates coefficients of the $i^{\text {th }}$ generator reflecting VPE.

Hence, the objective function for practical ELD issue can be devised in eq. (3).

$$
\mathrm{F}_{\mathrm{t}}=\sum_{\mathrm{i}=1}^{\mathrm{n}} \mathrm{F}_{\mathrm{i}}=\sum_{\mathrm{i}=1}^{\mathrm{n}} \mathrm{e}_{\mathrm{i}} \mathrm{p}_{\mathrm{gi}}^{2}+\mathrm{f}_{\mathrm{i}} \mathrm{p}_{\mathrm{gi}}+\mathrm{g}_{\mathrm{i}}+\left|\mathrm{a}_{\mathrm{i}} \times \sin \left(\mathrm{f}_{\mathrm{i}} \times\left(\mathrm{p}_{\mathrm{gi}}^{\min }-\mathrm{p}_{\mathrm{gi}}\right)\right)\right|
$$

Also, thermal generating units might supply multiple fuel types from diverse fuel sources. Every dispatching unit operates on multiple fuel sources depends on the load and the appropriateness of power generation. Both multiple fuel options and VPE ought to be represented to attain a realistic and high precise ELD solution. The cost function of $i^{\text {th }}$ generating units possessing VPE and multiple fuel choice is stated in eq. (4).

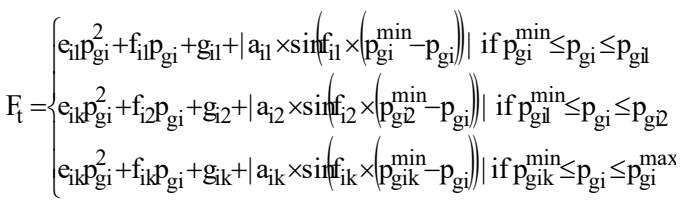

\subsection{Constraints}

The aforesaid objective function is stated as below constraints:

\subsubsection{Unit operating limit}

The active power generation of any unit must not go over its utmost and the least limits.

$$
\mathrm{p}_{\mathrm{gi}}^{\min } \leq \mathrm{p}_{\mathrm{gi}} \leq \mathrm{p}_{\mathrm{gi}}^{\max }
$$


In eq. (5), $\mathrm{p}_{\mathrm{gi}}$ indicates the number of the power output of $\mathrm{i}^{\text {th }}$ generator; $\mathrm{p}_{\mathrm{gi}}^{\max }$ and $\mathrm{p}_{\mathrm{gi}}^{\min }$ indicates the utmost and least power outputs of the $\mathrm{i}^{\text {th }}$ generator, correspondingly.

\subsubsection{Constraints of power balance}

The total generated power must be similar to total load demand and total losses and it is stated in eq. (6).

$$
\sum_{\mathrm{i}=1}^{\mathrm{n}} \mathrm{p}_{\mathrm{gi}}=\mathrm{p}_{\mathrm{D}} \leq \mathrm{p}_{\mathrm{L}}
$$

In eq. (6), $\mathrm{p}_{\mathrm{D}}$ represents the total system demand as well as $\mathrm{p}_{\mathrm{L}}$ represents total line loss. Transmission loss is stated in Kron's loss formula:

$$
\mathrm{p}_{\mathrm{L}}=\sum_{\mathrm{i}=1}^{\mathrm{n}} \sum_{\mathrm{i}=1}^{\mathrm{n}} \mathrm{p}_{\mathrm{gi}} \mathrm{B}_{\mathrm{ij}}+\sum_{\mathrm{i}=1}^{\mathrm{n}} \mathrm{B}_{0 \mathrm{i}} \mathrm{p}_{\mathrm{gi}}+\mathrm{B}_{00}
$$

In eq. (7), $B_{i j}$ represents $i-j^{\text {th }}$ element of loss coefficient of the symmetric matrix $B ; B_{0 i}$ represents $\mathrm{i}^{\text {th }}$ element of loss coefficient vector of the symmetric matrix $\mathrm{B} ; \mathrm{B}_{00}$ represents a coefficient constant of loss in normal operating circumstances.

\subsubsection{Prohibited operating zone}

Because of steam valve vibration or operation in shaft bearing, prohibited operating zones are contemplated. An optimal economy is attained by evading operation in such areas all over operation. The possible operating zones of $i^{\text {th }}$ unit are stated in eq. (8).

$$
\begin{aligned}
& p_{\mathrm{gi}}^{\min } \leq \mathrm{p}_{\mathrm{gi}} \leq \mathrm{p}_{\mathrm{gi}, 1}^{\mathrm{t}} \\
& \mathrm{p}_{\mathrm{gj}-1}^{\min } \leq \mathrm{p}_{\mathrm{gi}} \leq \mathrm{p}_{\mathrm{gi}, \mathrm{j}}^{\mathrm{t}} \quad \mathrm{j}=2,3, \ldots, \mathrm{n}_{\mathrm{i}} \\
& \mathrm{p}_{\mathrm{g} \mathrm{i}, \mathrm{ni}}^{1} \leq \mathrm{p}_{\mathrm{gi}} \leq \mathrm{p}_{\mathrm{gi}, 1}^{\max }
\end{aligned}
$$

In eq. (8), 1 and $\mathrm{u}$ indicates the upper and lower limits of a generation of a specific generator, $\mathrm{n}_{\mathrm{i}}$ indicates the number of prohibited zones for $\mathrm{i}^{\text {th }}$ generating unit.

\subsubsection{Ramp rate constraint}

Based on the generation maximization and minimization of generators, every generating unit is controlled by its RRL by superior units to operate frequently among 2 neighboring exact operation zones. These RRL constraints can be formulated in eq. (9).

$$
\max \left(p_{\mathrm{gi}}^{\min }, \mathrm{p}_{\mathrm{gi}}^{0}-\mathrm{DRL}_{\mathrm{i}}\right) \leq \mathrm{p}_{\mathrm{gi}} \leq \min \left(\mathrm{p}_{\mathrm{gi}}^{\max }, \mathrm{p}_{\mathrm{gi}}^{0}-\mathrm{URL}_{\mathrm{i}}\right)
$$

\section{Proposed Hybrid PSO-EBO Algorithm}

\subsection{PSO}

PSO is a population-based heuristic scheme for optimization issues [7]. It comprises a particle swarm, and every particle possesses a velocity and location in PSO. The position of a solution is recommended by the particle when velocity is change rate to the subsequent location regarding the current location. These two values are arbitrarily initialized, and solution model of PSO approach comprises two stages:

The velocity update of particle:

$$
\begin{aligned}
& u_{i}(t+1)=u_{i}(t)+c_{1} r_{1}\left(\text { pbest }_{i}-y_{i}\right)+c_{2} r_{2}\left(\text { gbest }_{i}-y_{i}\right) \\
& y_{i}(t+1)=y_{i}(t)+u_{i}(t+1)
\end{aligned}
$$

The location update of the Particle

$$
\mathrm{y}_{\mathrm{i}}(\mathrm{t}+1)=\mathrm{y}_{\mathrm{i}}(\mathrm{t})+\mathrm{u}_{\mathrm{i}}(\mathrm{t}+1)
$$

From (12), the particle velocity $\mathrm{i}$ is affected by 3 important modules such as the particle's old velocity $\mathrm{u}_{\mathrm{i}}(\mathrm{t})$; a linear magnetism towards personal optimal location ever found pbest $_{\mathrm{i}}-\mathrm{y}_{\mathrm{i}}$ scaled by the $\mathrm{c}_{1}$ weight and an arbitrary number $r_{1} \in[0,1]$; and the final module is a linear attraction towards global optimal location found by any swarm particle $\left(\right.$ gbest $\left._{\mathrm{i}}-\mathrm{y}_{\mathrm{i}}\right)$ scaled by weight $\mathrm{c}_{2}$ and an arbitrary number $\mathrm{r}_{2} \in[0,1]$. The particle's location for $t+1$ iteration is updated based on the eq. (11). 


\subsection{Bat Algorithm}

The BA is a bio-inspired approach [8] it is the basis of biosonar or echolocation features of microbats. The Movement of Artificial Bats and updating procedure of bat position is represented as below:

$$
\begin{aligned}
& \mathrm{f}_{\mathrm{i}}=\mathrm{f}_{\text {min }}+\left(\mathrm{f}_{\max }-\mathrm{f}_{\min }\right) * \beta_{\mathrm{i}} \\
& \mathrm{u}_{\mathrm{i}}(\mathrm{t}+1)=\mathrm{u}_{\mathrm{i}}(\mathrm{t})+\left(\mathrm{z}_{\mathrm{i}}(\mathrm{t}+1)-\mathrm{z}^{*}\right) \mathrm{f}_{\mathrm{i}} \\
& \mathrm{z}_{\mathrm{i}}(\mathrm{t}+1)=\mathrm{z}_{\mathrm{i}}(\mathrm{t})+\mathrm{u}_{\mathrm{i}}(\mathrm{t}+1)
\end{aligned}
$$

In eq. (13), $\beta_{\mathrm{i}}=\mathrm{t} * \mathrm{e}^{(-\rho * \mathrm{r})}, \mathrm{t}$ indicates iteration number, $\mathrm{r}$ indicates an arbitrary number $[0,1]$, the value of $\rho$ is an application dependent, for path planning issue value of $\rho$ is selected to be $(0.01)$. It represents $\beta$ raise with time for every bat producing minimum frequencies at previous phases of the search procedure. These frequencies augment about time to progress performance of the global search, whereas $z^{*}$ indicates current global optimal position solution, establish after creating an evaluation among all solutions between all $\mathrm{n}$ bats.

$$
\mathrm{z}_{\text {new }}=\mathrm{z}_{\text {old }}+\sigma \in \mathrm{A}(\mathrm{t})
$$

$\sigma$ indicates the scaling parameter comprises the step size control and $\mathrm{A}(\mathrm{t})$ indicates the average loudness of all bats at time step t. The $\mathrm{A}_{\mathrm{i}}$ represents the loudness, and pulse emission rate $\mathrm{r}_{\mathrm{i}}$, to be obliged to updated as iterations progress. Generally, loudness minimizes once a bat has established its prey, where pulse emission rate minimizes based on the formulations as stated below:

$$
\begin{aligned}
& A_{i}(t+1)=\alpha A_{i}(t) \\
& r_{i}(t+1)=r_{i}(0)[1-\exp (-\gamma t)]
\end{aligned}
$$

In eq. (17) and (18), $\alpha$ and $\gamma$ represents the design parameters.

\subsection{Developed Hybrid PSO-EBO Method}

To augment generally performance, benefit characteristics of two or additional optimization approaches are integrated to create a hybridized optimization approach. Here, hybridization among PSO and EBO approaches is developed. The variations of pulse emission rates $r_{i}$, loudness, $A_{i}$ also, present an auto zooming ability for optimization approach. To find optimum values of EBO method parameters $(\alpha, \gamma)$ is handled using the PSO approach. Nevertheless, such parameter settings can be issue-dependent accordingly difficult to describe. Additionally, the employ of time-varying parameters throughout such iterations can be beneficial. The appropriate control of such parameters able to, therefore, be significant, and as a result, differences of the parameters $\alpha$ as well as $\gamma$ (therefore the pulse rate $r_{i}$ and the loudness $\mathrm{A}_{\mathrm{i}}$ ) in an appropriate range is modified using the PSO approach to discover a balance among exploitation and exploration in EBA approach. In the proposed approach, the PSO solution is a vector of two dimensions.

\section{Results and Discussions}

\subsection{Experimental Procedure}

The experimentations were performed in diverse power generation systems to estimate developed method performance for several ELD issues having nonlinearities, like VPE, prohibited operating zone, and RRL. The developed method was tested and validated to exemplify the developed method performance.

\subsection{Performance Analysis}

Tables 1, 2, 3, and 4 summarizes the performance analysis of the proposed and conventional models such as GWO, ABC, WOA, and DE algorithms for Test systems 1,2 3, and 4. The developed method is examined and verified on 13, 40, 140 and160-unit systems to demonstrate the performance of the developed method. The overall analysis illustrates that the proposed method succeeds to find the best solution and worst cost' and 'mean cost' and minimum CPU time than conventional algorithms. 
Table 1: Performance analysis of proposed and conventional methods for test system 1

\begin{tabular}{lllllll}
\hline Algorithms & Best cost & Worst cost & $\begin{array}{l}\text { Mean } \\
\text { cost }\end{array}$ & $\begin{array}{l}\text { Standard } \\
\text { deviation }\end{array}$ & $\begin{array}{l}\text { Average computational timeSuccess } \\
\text { rate }\end{array}$ \\
\hline GWO & 26412.72 & 26418.09 & 26412.84 & 0.0988 & 4.16 & 96 \\
WOA & 26416.88 & 26416.88 & 26416.88 & - & 4.8 & - \\
DE & 26416.88 & - & 26416.81 & - & - & - \\
Proposed & 26460.06 & 26489.64 & 26461.66 & - & 10.6 & - \\
algorithm & & & & & & \\
\hline
\end{tabular}

Table 2: Performance analysis of proposed and conventional methods for test system 2

\begin{tabular}{llllllll} 
Algorithms & $\begin{array}{l}\text { Best } \\
\text { cost }\end{array}$ & Worst cost & Mean cost & $\begin{array}{l}\text { Standard } \\
\text { deviation }\end{array}$ & $\begin{array}{l}\text { Average computational } \\
\text { time }\end{array}$ & $\begin{array}{l}\text { Success } \\
\text { rate }\end{array}$ \\
\hline GWO & 136440.6 & 136446 & 136442.3 & 0.1003 & 10.23 & 96 \\
ABC & 136446.9 & 136492.1 & 136464 & 0.1282 & 11.06 & 90 \\
WOA & 137329.9 & 136671.9 & 136671.2 & - & 10.7 & - \\
DE & 136670.4 & 136671.9 & 139865 & - & 14 & - \\
Proposed & 139865 & - & - & - & - & - \\
algorithm & & & & & & \\
\hline
\end{tabular}

Table 3: Performance analysis of proposed and conventional methods for test system 3

\begin{tabular}{|c|c|c|c|c|c|c|}
\hline Algorithms & $\begin{array}{l}\text { Best } \\
\text { cost }\end{array}$ & Worst cost & Mean cost & $\begin{array}{l}\text { Standard } \\
\text { deviation }\end{array}$ & $\begin{array}{l}\text { Average computational } \\
\text { time }\end{array}$ & $\begin{array}{l}\text { Success } \\
\text { rate }\end{array}$ \\
\hline GWO & 2559710 & 2559733 & 2559723 & 0.0936 & 32.77 & 99 \\
\hline ABC & 2559953 & 2560229 & 2560233 & 0.223 & 35.52 & 90 \\
\hline WOA & 2559305 & 2559306 & 2559306 & - & 36.9 & - \\
\hline DE & 2560237 & - & - & - & - & - \\
\hline $\begin{array}{l}\text { Proposed } \\
\text { algorithm }\end{array}$ & 2559710 & 2559733 & 2552723 & 0.0936 & 32.77 & 99 \\
\hline
\end{tabular}

Table 4: Performance analysis of proposed and conventional methods for test system 4

\begin{tabular}{|c|c|c|c|c|c|c|}
\hline Algorithms & Best cost & Worst cost & Mean cost & $\begin{array}{l}\text { Standard } \\
\text { deviation }\end{array}$ & $\begin{array}{l}\text { Average } \\
\text { computational } \\
\text { time }\end{array}$ & Success rate \\
\hline GWO & 9755.113 & 9757.215 & 9756.1 & 0.0996 & $\begin{array}{ll}6 & 16.32\end{array}$ & 97 \\
\hline $\mathrm{ABC}$ & 9713.377 & 9719.573 & 9715.533 & 0.1271 & 17.65 & 92 \\
\hline WOA & 9971.974 & 9973.997 & 9972.991 & - & 17.3 & - \\
\hline DE & $10,005.20$ & $10,005.55$ & $10,005.21$ & - & 19 & - \\
\hline $\begin{array}{l}\text { Proposed } \\
\text { algorithm }\end{array}$ & $10,007.71$ & $10,010.59$ & $10,009.16$ & - & 55 & - \\
\hline
\end{tabular}

\section{Conclusion}

In this work, the implementation of a hybrid PSO-EBO approach was developed. The hybrid PSO-EBO was selected against conventional optimization algorithms to resolve several non-linear Economic Load Dispatch issues for superior convergence rate and sturdiness to produce superior possible solutions. It was renowned that PSO was the capable and main easy optimization algorithms that demonstrate its advantage in diverse fields of optimization issues. To improve the search effectiveness of PSO was combined with the EBO algorithm. To show the possibility and the effectiveness of the developed technique, 4 numerical experimentations were to resolve large, small, and medium-scale ELD issues. Several nonlinearities such as prohibited operating zone, VPE, and RRL were considered in the simulations. Finally, the outcomes of the experimentation exhibit the advantage of the developed method over other various well-known algorithms. The improved computation effectiveness, strength, and convergence of the developed algorithm demonstrate that the developed algorithm might have experimented in several regions of optimization issues.

\section{References}

[1] Diljinder Singh, J. S. Dhillon,"Ameliorated grey wolf optimization for economic load dispatch problem",Energy,Volume 16915, February 2019,Pages 398-419. 
[2] Maedeh Gholamghasemi, Ebrahim Akbari, Mohammad Bagher Asadpoor, Mojtaba Ghasemi,"A new solution to the non-convex economic load dispatch problems using phasor particle swarm optimization", Applied Soft Computing,Volume 79,June 2019,Pages 111-124.

[3] Mohit Kumar, J. S. Dhillon,"Hybrid artificial algae algorithm for economic load dispatch",Applied Soft Computing,Volume 71,October 2018,Pages 89-109.

[4] S. Hr. Aghay Kaboli, Abdullah K. Alqallaf,"Solving non-convex economic load dispatch problem via artificial cooperative search algorithm",Expert Systems with Applications,Volume 128,15 August 2019,Pages 14-27.

[5] Qiang Zhang, Dexuan Zou, Na Duan, Xin Shen,"An adaptive differential evolutionary algorithm incorporating multiple mutation strategies for the economic load dispatch problem",Applied Soft Computing,Vol. 78,May 2019,pp 641-669.

[6] Yizi Shang, Shibao Lu, Jiaguo Gong, Ronghua Liu, Qixiang Fan,"Improved genetic algorithm for economic load dispatch in hydropower plants and comprehensive performance comparison with dynamic programming method",Journal of Hydrology,Volume 55,4November 2017,Pages 306-316.

[7] R.C. Eberhart, J. Kennedy, R.C. Eberhart, Particle swarm optimization, Proc.IEEE Int. Conf. Neural Networks IV, pp. 4 (1995) 1942-1948.

[8] X.S. Yang, A new metaheuristic Bat-inspired Algorithm, in: Stud. Comput.Intell., 2010: page no. 65-74.

[9] Raj Kumar",Hybrid Cat Swarm and Crow Search Algorithm to Solve the Combined Economic Emission Dispatch Model for Smart Grid",Journal of Computational Mechanics, Power System and Control (JCMPS), Volume 2, Issue 3, July 2019.

[10] Chithra.S and R.Madahana Kumari,"Economic Emission Dispatch in Renewable Energy Systems using FireFly Algorithm",Journal of Computational Mechanics, Power System and Control (JCMPS), Volume 1, Issue 1, October 2018.

[11] Rekha .P. Nair,Kanakasabapathy P,"Hybrid PSO-BF Algorithm for Economic Dispatch of a Power System",Journal of Computational Mechanics, Power System and Control (JCMPS), Volume 2, Issue 2, April 2019. 